\title{
REVIEW
}

\section{New Horizons in MR Technology: RF Coil Designs and Trends}

\author{
Hiroyuki FuJITA ${ }^{1,2,3 *}$ \\ Departments of ${ }^{1}$ Physics, Case Western Reserve University, and ${ }^{2}$ Radiology, University \\ Hospitals of Cleveland, 10900 Euclid Avenue, Cleveland, Ohio 44106-7079, USA \\ ${ }^{3}$ School of Information Technology and Electrical Engineering, \\ The University of Queensland, Brisbane Qld 4072, Australia
}

(Received January 9, 2007; Accepted February 14, 2007)

\begin{abstract}
Parallel imaging techniques have developed very rapidly, and realization of their full potential has required the design of magnetic resonance (MR) scanners with ever-increasing numbers of receiver channels (32 to 128). In particular, 1.5- and 3-Tesla fast MR imaging applications are now used in everyday clinical practice. Both strengths require maximum achievable signal-to-noise ratio (SNR) and multi-detector array coil optimization within the framework of the parallel imaging scheme for more advanced and faster clinical MR scanning. Preamplifiers are key components in the detector array coils and serve many functions beyond mere signal amplification. One critical function is to aid in the decoupling of individual coils, which is essential for optimal SNR and the performance of parallel imaging. To support a large number of detector array coils for parallel imaging, preamplifiers must be physically very small so that they may be tightly packed together to form an optimized detector array. The author herein reviews the state-of-the-art work reported by those skilled in the art to consider the rationale for determining how many channels are enough and how fast we can go. The paper explores the important and fundamental principles of RF array coils for MR imaging and reviews cutting-edge array coils, including those for transmit-SENSE or parallel transmission applications. The future of radiofrequency (RF) coil technology is also considered.
\end{abstract}

Keywords: phased array $R F$ coils, preamplifier decoupling, parallel imaging, parallel transmission, wireless $R F$ coils

\section{Introduction}

Modern radiofrequency (RF) array coil design is complex, and its understanding requires broad knowledge of magnetic resonance (MR) physics and electronics. Today's coils are very intricate and delicate. For example, as many as 400 to 1500 individual components/parts are used in the construction of 8-channel head coils and 16-channel brain-spine combo coils, both of which are typical detector array coils. Every component must be correctly integrated; if even one component is broken or damaged, the coil does not function optimally. The author attempts to explain the critical components that constitute an RF array coil, especially emphasizing principles and methods for decoupling array coils, which is essential to

*Corresponding author, Phone: +1-216-368-3894, Fax:+1216-368-4671, E-mail: hiroyuki.fujita@case.edu today's advanced and sophisticated clinical applications.

In 1978, in his historic paper, Hoult detailed and discussed the nuclear magnetic resonance (NMR) receiver ${ }^{1}$ in 1990 , Roemer and associates proposed the phased-array coil technology that underlies the design of most of today's RF receive coils; ${ }^{2}$ and in 1991, Hayes's group discussed the application of that technology to volume imaging. ${ }^{3}$ Original and general phased-array radar technology ${ }^{4,5}$ and array coil theory and application ${ }^{6}$ are reviewed in detail elsewhere.

A small surface coil is well known to yield higher SNR nearer the surfaces of the coil than does a volume coil, such as the birdcage coil, ${ }^{7}$ but the $\mathrm{B}_{1}$-sensitive region of a small surface coil is (much) smaller than that of a volume coil. It is remarkable that in an approach utilizing phased-array coils, the high signal-to-noise ratio (SNR) associated with a small surface coil can be achieved and maintained 


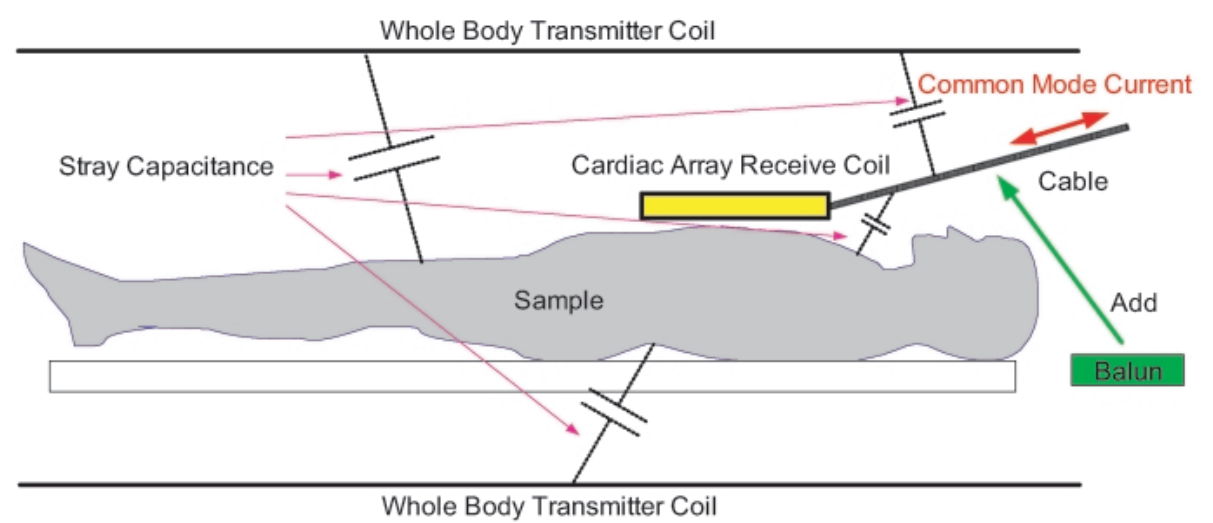

Fig. 1. The mechanism of electromagnetic coupling between the patient and the magnetic resonance (MR) radiofrequency (RF) coils, due to the stray capacitances present, is illustrated. Baluns are added to block the common mode current and allow the differential mode current to flow.

over an extended field of view (FOV) by using a set of RF coils. This is possible because of the development of various array coil designs for imaging applications of interest and of techniques to decouple the mutual inductances and thereby eliminate cross-talk among the elements of the array coils. Adjacent coil elements are often partially overlapped to cancel the mutual inductance between the elements. The next nearest and other coil elements can be mutually decoupled using a low-input impedance (typically less than $5 \mathrm{Ohms}$ ) preamplifier decoupling circuit. Other decoupling techniques and methods are briefly discussed later; detailed discussions of low noise amplifiers may be found elsewhere. ${ }^{8}$

In conjunction with a coil matching/decoupling circuit, the low-input impedance preamplifier effectively eliminates current flow in the coil element loop, thereby eliminating the magnetic fields induced in neighboring coil elements (shown later). Baluns and considerations pertaining to cable routing are also important factors in array coil design. Figure 1 illustrates how cables couple with the patient and why baluns need to be implemented; details are discussed elsewhere. ${ }^{9}$

RF coil technologies are driven by the rapid advances in development of MR scanners with greater numbers of receiver channels as well as in development of parallel imaging techniques; $;^{10-12}$ the 96-channel head array coil is one such example. ${ }^{13}$ Whatever the application at any field strength, maximum achievable SNR is key. Because SNR is destined to be degraded or lowered from the starting point, i.e., the signal source, to the end point of the receiver chain, minimizing its loss in the process is of primary concern. In this paper, the components of an array coil are explained, and issues requiring special attention are noted. The author uses state-of-the-art examples to address the questions of what constitutes a sufficient number of channels and how coil elements in an array are decoupled and to consider how array detectors can be optimized.

\section{Signal-to-Noise Ratio}

Signal-to-noise ratio (SNR), one of the most important parameters to be optimized in MR imaging applications, was detailed by Hoult and Richards. ${ }^{14}$ Here, it is essential to understand how SNR relates to the coil-related parameters. The signal is written as

$$
S \propto \gamma^{3} B_{0}^{2} B_{1}^{x y}(r) \quad \text { Eq. (1), }
$$

where $\gamma$ is the nuclear gyromagnetic ratio, $\mathrm{B}_{0}$ is the static magnetic field, and $B_{1}{ }^{x y}(r)$ is the $R F$ magnetic field produced by a coil with unit current $1 \mathrm{~A}$. In designing a coil, $\mathrm{B}_{1}{ }^{\mathrm{xy}}(\mathrm{r})$ must be optimized, which requires the appropriate choices of coil size over a target FOV and of distance from the FOV. Size choice depends generally on the number of available receiver channels.

On the other hand, NMR/MR imaging noise is thermal noise, and the noise generated from the coil is given by

$$
N=\sqrt{4 k T \Delta f R}
$$

where $\mathrm{k}$ is the Boltzmann constant, $\mathrm{T}$ is the temperature in $\mathrm{K}, \Delta \mathrm{f}$ is the bandwidth, and $\mathrm{R}=\mathrm{R}_{\mathrm{C}}+\mathrm{R}_{\mathrm{S}}$. $R_{C}$ is the coil resistance and $R_{S}$ the sample energy loss, i.e., the equivalent series resistance resulting from the induced eddy current losses in the conductive sample. Combining Eqs. (1) and (2), SNR can be expressed using the coil-related parameters as 
Table 1. Relationship between $\mathrm{dB}$ expression and common factor

\begin{tabular}{cccccccccccccc}
\hline Factor & $1 / 100$ & $1 / 10$ & $1 / 5$ & $1 / 3$ & $1 / 2$ & 2 & 3 & 4 & 5 & 6 & 7 & 10 & 100 \\
\hline $\mathrm{dB}$ & -20 & -10 & -7 & -4.8 & -3 & 3 & 4.8 & 6 & 7 & 7.8 & 8.45 & 10 & 20 \\
\hline
\end{tabular}

$$
\frac{S}{N} \propto \frac{B_{1}^{x y}(r)}{\sqrt{R_{C}+R_{S}}}
$$

Eq. (3).

To optimize SNR, $B_{1}{ }^{\mathrm{xy}}(\mathrm{r})$ can be maximized by having the coil closer to the sample, and $\mathrm{R}_{\mathrm{S}}$ can be minimized by matching coil size to the target FOV. In fact, Wright and Wald compared an N-element array to a single coil with respect to the resultant SNR while overall coil dimension remained unchanged. ${ }^{6}$ They showed, for example, that when comparing a single coil with arrays measuring $8 \times 8$, $4 \times 4$, and $2 \times 2$, improvement in SNR disappears at a depth equal to the diameter of the array. However, for distances in between, SNR curves vary according to the number of array (i.e., coil) elements. If each coil is made too small, the coil resistance loss dominates the sample noise. This implies that optimization is required for a given specific application, e.g., target depth and available number of receiver channels. $R_{C}$ is minimized by making the unloaded coil Q high. It is well known that at low- $\mathrm{B}_{0}$ field systems $(\leq 0.3 \mathrm{~T})$, coil resistance is dominant or at least comparable to sample noise, but at $1.5 \mathrm{~T}$ and above, sample noise dominates (i.e., $R_{S} \gg R_{C}$ ). Furthermore, it is useful to be aware that if each of 2 coils yields the same relative sensitivity in free space and is dominated by sample noise, the two have the same absolute sensitivity. ${ }^{15}$ (The detailed discussion of the coil unloaded Q, loaded Q, and sensitivity in this reference should be useful to the coil engineer.)

\section{Low Noise Preamplifier}

The characteristic parameters of preamplifiers, such as the noise figure (NF), affect SNR performance. Thus, a preamplifier is a key component in an RF coil and plays a critical role in the design of a detector array coil. A brief summary of the preamplifier's function follows. The electromotive force or induced voltage (i.e., signal) in a coil is very small, typically on the order of a few $\mu \mathrm{V}$. A preamplifier increases this small signal to a few $\mathrm{mV}$, e.g., $30 \mathrm{~dB}$ (i.e., 1000 times) greater. In practice, gain usually refers to power gain; thus, a $20-\mathrm{dB}$ gain in power corresponds to a $10-\mathrm{dB}$ gain in voltage. (Quick conversion factors are shown in Table 1.)

The NF, one parameter for measuring the performance of the preamplifier, is detailed else-



Fig. 2. Depiction of a preamplifier noise model. V denotes the signal; $\mathrm{N}_{\mathrm{S}}$, the noise generated by the resistance, $r$, of the input signal source; and $\mathrm{N}_{P}$, the noise generated inside the preamplifier.

where. ${ }^{1,16}$ Figure 2 depicts a simple model for preamplifier noise. $\mathrm{V}$ denotes the signal; $\mathrm{N}_{\mathrm{S}}$, the noise generated by the resistance, $r$, of the input signal source; and $\mathrm{N}_{P}$, the noise generated inside the preamplifier. Using these quantities, NF is defined as

$$
N F=10 \log _{10}\left(\frac{N_{P}^{2}+N_{S}^{2}}{N_{S}^{2}}\right) \quad \text { where } \quad N_{S}=\sqrt{4 k T \Delta f r}
$$

The industry standard for preamplifier NF is less than $0.5 \mathrm{~dB}$, and the first NF and gain are well known to have the most significant impact in the entire electronics circuit, which is often cascaded. Kucera, Lott, Horowitz and Hill demonstrate this in their respective chapters on the design of lownoise amplifiers. ${ }^{8,16}$ Because noise is generated in any passive elements that dissipate power, including cables, the design of most detector array coils integrates preamplifiers so as to minimize undesirable noise. The multi-detector array is a key component for parallel imaging and comprises many (32 to 128) detectors. As stated, preamplifiers not only amplify signals; they are key features of these detectors that assist in the decoupling of individual detectors, and decoupling is critical to optimizing parallel imaging. The very small size of the preamplifiers allows them to be tightly packed to form an optimized array.

\section{A Single Coil Model}

To understand the mechanism underlying the function of detector array coils, we start with a schematic representation of a single coil that includes a simplified preamplifier decoupling circuit (Fig. 3[a]). This coil circuit is a building block of an array coil. $\mathrm{L}_{1}$ represents the coil inductance and 
$\mathrm{R}_{1}$, the coil resistance (typically around $0.5 \mathrm{Ohms}$ in air and 5 Ohms when placed on a phantom). $C_{1}$ and $\mathrm{C}_{2}$ are the respective tuning and matching capacitances. When looked at from the side of the preamplifier (i.e., that side of the circuit), a part of $\mathrm{L}_{2}$, a matching inductor, functions to match the coil impedance to $50 \mathrm{Ohms}$ together with $\mathrm{C}_{2}$. At the same time, $\mathrm{L}_{2}$ achieves a parallel resonant circuit formed with $\mathrm{C}_{2}$, in particular, when the input impedance of the preamplifier, $r_{\text {preamp }}$, becomes small (e.g., 0 to $2 \mathrm{Ohms}$ ). When looked at from the side of the coil, impedance is infinity, so the circuit has high impedance and thus is equivalently open. It is extremely important to note that because current cannot flow in an open coil, no magnetic field can be induced through non-zero mutual inductance in neighboring coil elements. This principle underlies the elimination of cross-talk among coil elements. This decoupling mechanism is explained in more detail later, but it is worth mentioning that the original signal source changes from the current source (without preamplifier decoupling) to the voltage source (with preamplifier decoupling) that contains the necessary information without losing the integrity of the original signal information.

\section{Preamplifier Decoupling}

We now consider a system of 2 coupled coils and the underlying mechanism of their decoupling. In Fig. 3(b), Coils 1 and 2 are coupled through mutual inductance, $M_{12}$. Coil 1(2) is represented by its selfinductance, $\mathrm{L}_{1(2)}$; resistance, $\mathrm{R}_{1(2)}$; and capacitance $\mathrm{C}_{1(2)} . \mathrm{V}_{\text {signal }}$ indicates the signal induced in Coil 1, and $\mathrm{V}_{\text {out }}$ indicates the output voltage. When the $\mathrm{AC}$ current, $\mathrm{I}_{1}$, flows in Coil 1 , the induced AC current, $\mathrm{I}_{2}$, will flow in Coil 2 through the non-zero mutual coupling between the 2 coils. Thus, assuming harmonic time dependence (i.e., $e^{-i \omega t}$ ), the output voltage is expressed as

$$
V_{\text {out }}=V_{\text {signal }}+\left(R_{1}+i\left(\omega L_{1}-\frac{1}{\omega C_{1}}\right)\right) I_{1}+i \omega M_{12} I_{2}
$$

This equation is essential to understand the need for the coil to be resonated at the target Larmor frequency and to comprehend the mechanism of preamplifier decoupling. The second term in the right side in Eq. (5) associated with $\mathrm{I}_{1}$ may be realized as the noise related to Coil 1 , and the third term associated with $I_{2}$ is recognized as the noise resulting from the coupling between Coils 1 and 2 . The noise related to Coil 1 can be minimized by tuning and matching Coil 1 for resonance, i.e.,

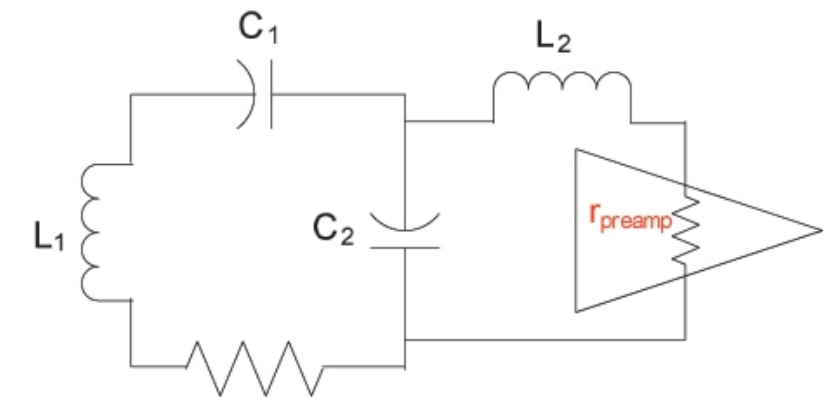

a

$\mathrm{R}_{1}$
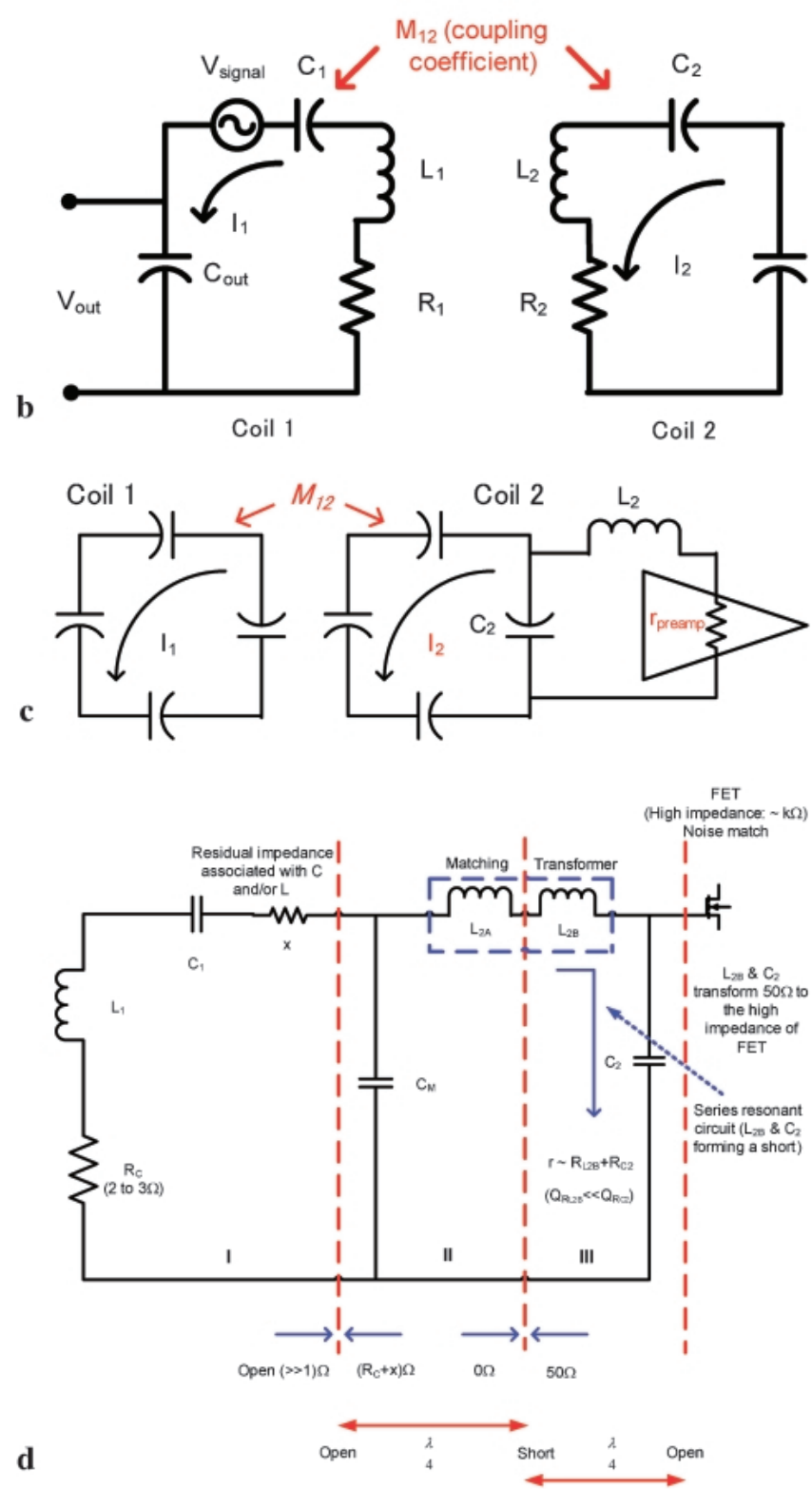

Fig. 3. (a) A single-coil model with a low-input impedance preamplifier. (b) A system of 2 coils coupled through non-zero mutual inductance. (c) A system of 2 coils coupled through non-zero mutual inductance. A low-input impedance preamplifier is added to Coil 2 for preamplifier decoupling. (d) The matching inductor $\mathrm{L}_{2}$ $\left(\mathrm{L}_{2 \mathrm{~A}}+\mathrm{L}_{2 \mathrm{~B}}\right)$ plays several critical functions in preamplifier decoupling. Each quarter- $\lambda$ circuit has its characteristic impedance for necessary impedance matching. 
the imaginary part of the term associated with $\mathrm{I}_{1}$ vanishes, leaving the intrinsic real resistance, $R_{1}$. Decoupling requires that the third term in the right side in Eq. (5), $i \omega M_{12} I_{2}$, i.e., the noise through the mutual coupling, be zero. To satisfy this condition, we can arrive at 2 cases: either $M_{12}=0$ or $I_{2}=0$. When $M_{12}=0$, Coils 1 and 2 are overlapped to nullify the mutual inductance between the two. Capacitive decoupling may be employed here to cancel the mutual inductance. ${ }^{17}$ The principle behind the capacitive decoupling is that the nonzero mutual impedance is modeled as an inductance, and the inductance is canceled by the addition of capacitance (i.e., the 2 coils are connected by a capacitor). $I_{2}=0$ corresponds to the case of so-called preamplifier decoupling.

Now Coil 2 is integrated with a preamplifier decoupling and matching circuit (Fig. 3[c]). Here, $\mathrm{L}_{2}$ is a matching inductor, and $\mathrm{r}_{\text {preamp }}$ represents the input impedance of the preamplifier; as $\mathrm{r}_{\text {preamp }}$ becomes 0 , the inductor, $\mathrm{L}_{2}$, and the capacitor, $\mathrm{C}_{2}$, can be chosen to form a parallel resonant circuit at the target MR frequency, yielding a high impedance (i.e., infinity, theoretically speaking). Coil 2 then becomes an open circuit, and there is no current flow, i.e., $\mathrm{I}_{2}=0$. At this stage, even if the mutual coupling between the 2 coils is not zero, Coils 1 and 2 are decoupled using a low-input impedance preamplifier. This is the art of preamplifier decoupling. Furthermore, it is emphasized that the matching inductor, $\mathrm{L}_{2}$, denoted as $\mathrm{L}_{2 \mathrm{~A}}$ and $\mathrm{L}_{2 \mathrm{~B}}$ in Fig. 3(d), plays several crucial functions that may not appear obvious. $\mathrm{L}_{2 \mathrm{~A}}$ functions to match the coil impedance to $50 \mathrm{Ohms}$ together with $\mathrm{C}_{M}$ when looked at from the side of the preamplifier $(\mathrm{III} \rightarrow \mathrm{II})$. At the same time, $\mathrm{L}_{2 \mathrm{~A}}$ (and $\mathrm{L}_{2 \mathrm{~B}}$ and $\mathrm{C}_{2}$, which are adjusted to be "short," i.e., the series resonance) achieves a parallel resonant circuit formed with $\mathrm{C}_{\mathrm{M}}$, in particular, when the input impedance of the preamplifier, $r_{\text {preamp }}$ (approximately $\mathrm{R}_{\mathrm{L} 2 \mathrm{~B}}+\mathrm{R}_{\mathrm{C} 2}$ ), becomes small (e.g., 0 to $2 \mathrm{Ohms}$ ). When looked at from the side of the coil (I $\rightarrow$ II), the impedance is infinity, thereby having a highimpedance or open circuit. In other words, the decoupling circuit considered here and shown in Fig. 3(d) (sandwiched by 2 dashed lines) is equivalently a mismatched $\lambda / 4$ (quarter wavelength cable) circuit; one end is open and the other end is short. It is instructive and meaningful to view the circuit (regions denoted as II and III) such that each region may be represented as a corresponding $\lambda / 4$ circuit with its characteristic impedance, $Z_{0}$. A transmission line that is an odd multiple of quarter wavelengths long that terminates in an impedance $\mathrm{Z}_{\text {Load }}$ presents an input impedance of

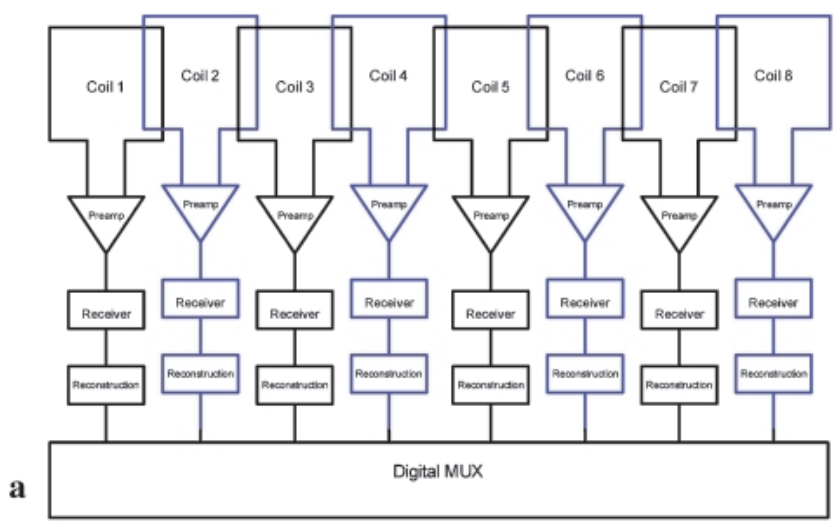

b

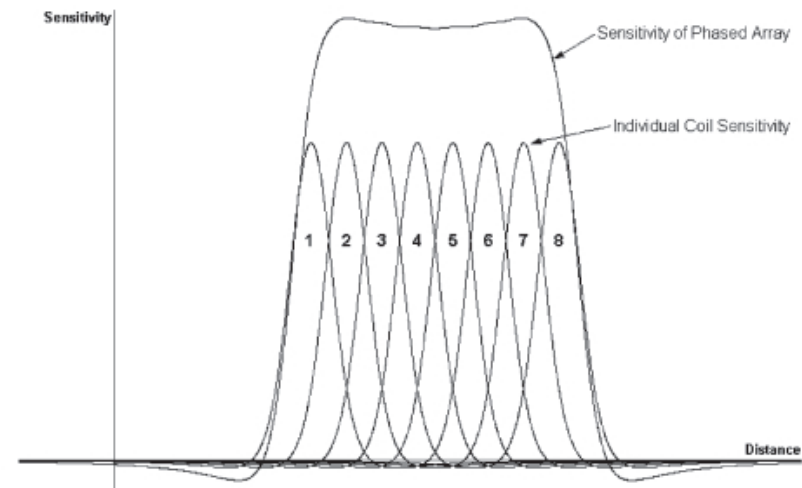

Fig. 4. (a) Schematic representation of a detector array coil (8-channel). (b) Depiction of $\mathrm{B}_{1}$ sensitivity profile in an 8-channel array coil in arbitrary units.

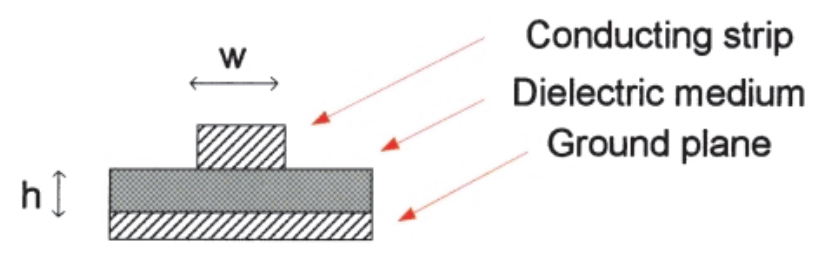

\section{Microstrip line}

Fig. 5. The structure of a micro-strip line coil. To achieve necessary impedance matching, the thickness, $h$, of the dielectric medium and the width, $w$, of the conducting strip are adjusted such that the characteristic impedance $\mathrm{Z}_{0}=50 \Omega$.

$$
Z_{\text {in }}=\frac{Z_{0}^{2}}{Z_{\text {Load }}}
$$

where $Z_{0}=$ characteristic impedance. In turn, $Z_{0}=$

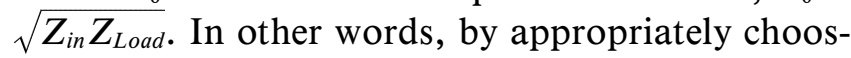
ing the characteristic impedance of the matching section, a quarter-wave section can be used to match any 2 impedances, such as in sections II and III of Fig. 3(d). The transformations in impedance from I to II to III and in reverse are executed correctly and consistently because each region has 
its associated and appropriate characteristic impedance. With this quarter-wave matching section mechanism, we turn to the preamplifier.

A preamplifier is noise matched; for a given fieldeffect transistor (FET), there is a signal-source impedance that optimizes or minimizes the noise figure. The optimum NF can be achieved when the impedance of the receive coil matches the source impedance of the FET. Because the FET impedance is relatively high ( $\gg 50 \mathrm{Ohms}$ ), an impedance transformer comprising $\mathrm{L}_{2 \mathrm{~B}}$ and $\mathrm{C}_{2}$ together forms a $\lambda / 4$ circuit in section III of Fig. 3(d) and transforms $50 \mathrm{Ohms}$ to the high input impedance of the FET for the lowest NF. An optimal impedance for the lowest noise figure of typical FET is on the order of a few hundred $\Omega$. Thus, $\mathrm{L}_{2 \mathrm{~B}}$ and $\mathrm{C}_{2}$ transform the coil's $50 \mathrm{Ohms}$ to the high impedance of FET for the lowest NF. As an additional note, the input impedance of the preamplifier in Fig. 3(d) is approximated as the sum of resistances associated with $\mathrm{L}_{2 \mathrm{~B}}$ and $\mathrm{C}_{2}$. However, since the $\mathrm{Q}$ of the capacitor is typically much higher than that of the inductor, the input impedance is dominated by the contribution of the resistance associated with the inductor.

With an understanding of this preamplifier decoupling method, it is straightforward to extend the array concept to 8-, 16-, and even larger numbers of channels. Figure 4(a) shows an 8-channel array coil in which each coil has its own $B_{1}$ sensitivity profile (Fig. 4[b]). Because it is a surface coil, which yields a high SNR over only a small region, an overall high SNR over the target FOV or region of interest (ROI; Fig. 4[b]) can be generated if each channel SNR is combined by a sum-of-squares method, ${ }^{2}$ for example. As discussed, taking into consideration the appropriate size of each coil with SNR optimization, the number of coil channels is determined by the number of available receiver channels and the target FOV or ROI. Many references discuss different array coil designs for various imaging applications at different field strengths. Although a detector array coil using quadrature pairs (for each channel) is possible, as the number of available receiver channels increases, the current trend is to use a set of various (size and shape) loop coils.

\section{Other Array Coil Designs}

This paper focuses on detector array coil designs that employ preamplifier decoupling because these are the most popular and widely accepted designs at 1.5 and 3 Tesla. Other decoupling techniques include: overlap; ${ }^{2}$ quadrature (intrinsic isolation/ decoupling); solenoidal array employing antiturn loop; ${ }^{18}$ capacitor decoupling to cancel inductance ${ }^{17,19}$ decoupling network after coils to manipulate the electric field induced by the mutual inductive coupling $;{ }^{20}$ and shield or transmission line designs, which are often employed for very high field applications (Fig. 5) because most electromagnetic fields are contained between the coil element and the shield..$^{21,22}$ In these shielded or transmission line designs, for imaging, electromagnetic-field leakage is small, and because the leaked fields are only a small portion of all fields, the isolation between coils remains adequate even if leaked electromagnetic fields couple with each other.

\section{Various Parallel Imaging Methods (SMASH versus SENSE)}

With the advent of parallel imaging techniques, ${ }^{10-12,23}$ array coil design plays a major role in achieving desired parallel-imaging performance. ${ }^{24-33}$ However, with respect to coil design, there is a major difference between simultaneous acquisition of spatial harmonics (SMASH), a "k-space" reconstruction approach, and sensitivity encoding (SENSE), an "x-space" (i.e., image domain) reconstruction approach. In SMASH, spatial harmonics are achieved by manipulating the $B_{1}$ sensitivity of each coil in the array to replace some k-space data lines that would otherwise have been collected by the application of conventional gradient phase encoding. This requires that the directions of the array and desired phase encoding coincide. In clinical practice, the complex shapes and curvature of the body often make it difficult to construct a SMASH array coil whose direction/ orientation coincides with that of the desired phase encoding, at the same time generating the spatial harmonics required in that direction. In contrast, SENSE is less restrictive about placement of each channel coil in conjunction with a chosen phase encoding direction. However, SENSE requires that one be able to tell which $B_{1}$ sensitivity belongs to which coil so that the aliased image that is created unfolds and the desired acceleration factor can be achieved. The acceleration factor is the inverse of the reduction factor in imaging time. The measure of ease to distinguish $\mathrm{B}_{1}$ sensitivity is given by a so-called g-factor, and for a given imaging application, coil design requires that the g-factor be optimized. ${ }^{34}$ Furthermore, the acceleration factor depends upon the number of receiver channels; e.g., to achieve an acceleration factor of 3 in $\mathrm{x}$ direction, there must be at least 3 individual coils in that direction. Additionally, Ohliger and associates 
a
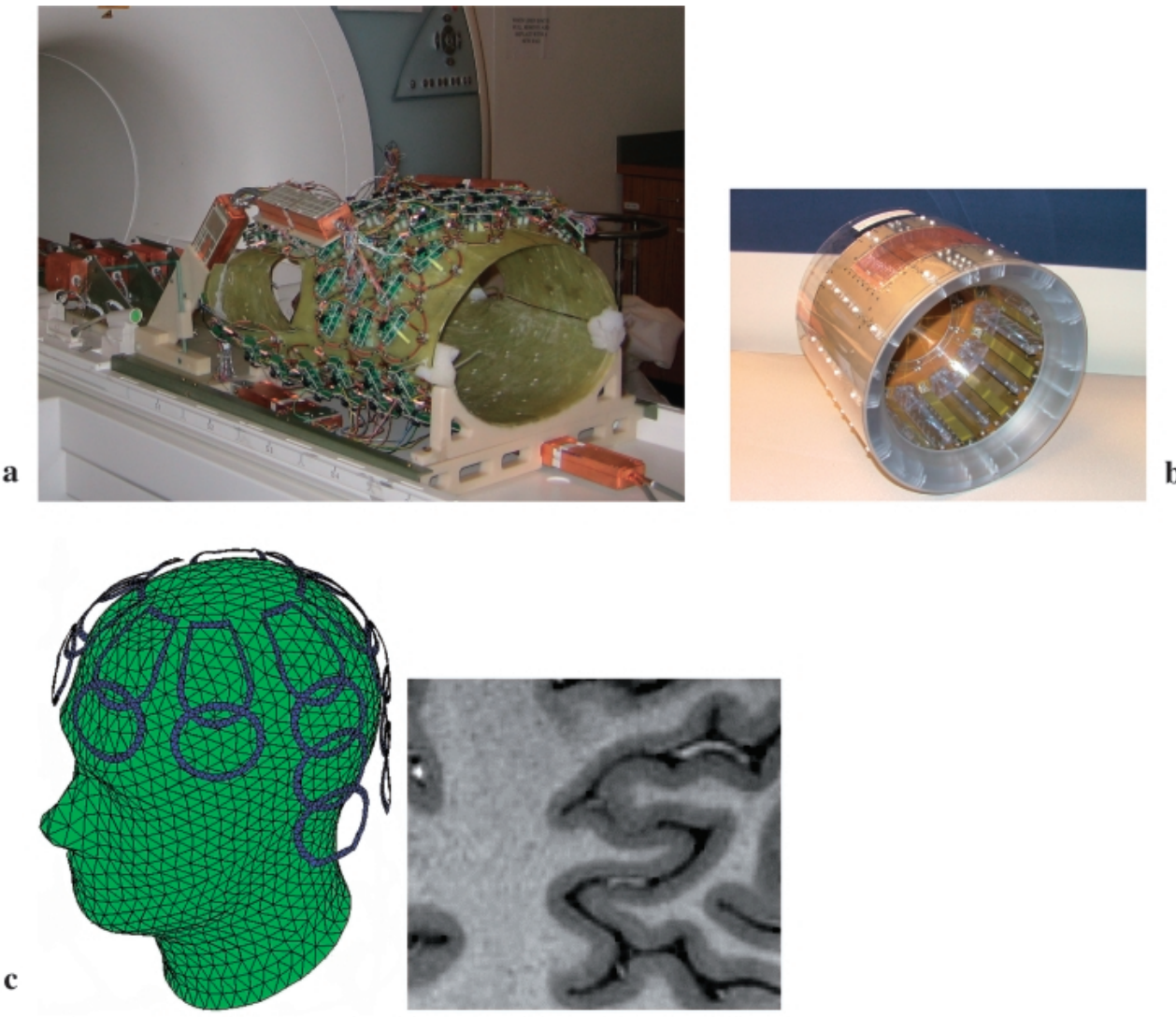

b

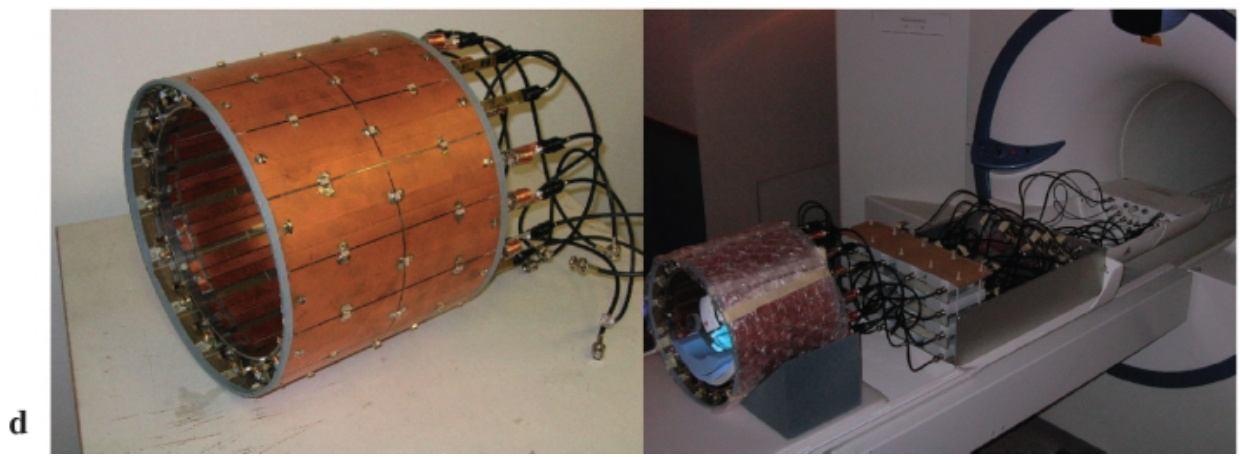

Fig. 6. (a) Prototype coil of 3T, 128-channel cardiac array coil. (Courtesy Lawrence L. Wald, Massachusetts General Hospital). (b) A 7T base shielded birdcage $T / R$ brain coil measuring $18 \mathrm{~cm}$ long with a diameter of $27 \mathrm{~cm}$. The shield protects against radiation loss at very high frequencies. (Courtesy John L. Patrick, Philips Medical Systems). (c) A 7T, 24-channel whole-brain array. Twenty-four elements are arranged in 8 radially gapped columns (30\% gap between elements). The array has anterior columns ( 2 elements) and posterior columns (4 elements). The image is obtained with MPRAGE: repetition time/ echo time (TR/TE) $5 / 11 \mathrm{~ms} ; 512 \times 512 ; 2-\mathrm{mm}$ slice; $0.5 \times 0.5 \times 2 \mathrm{~mm}^{3}$ voxels. (Courtesy Patrick J. Ledden, Nova Medical, and Jeff H. Duyn, National Institutes of Health [NIH], Bethesda, MD). (d) A 7T, 16-channel volume strip array with 16-way T/R interface. (Courtesy Ray F. Lee, New York University).

attempted to establish an upper bound on the spatial encoding capabilities of array coils in parallel imaging. ${ }^{35}$ A few highlights from their work include that: a limit for acceleration is $5 \times$ for 1D undersampling of $\mathrm{k}$-space; a net acceleration factor of $15 \times$ is the limit for $2 \mathrm{D}$; a higher accelera- tion factor can be achieved as $\mathrm{B}_{0}$ becomes higher (due to shorter wavelength); and tissue conductivity affects parallel imaging performance. The achievable SNR with finite coil arrays is discussed in. ${ }^{36}$ These insightful considerations should be helpful to coil designers/engineers. 

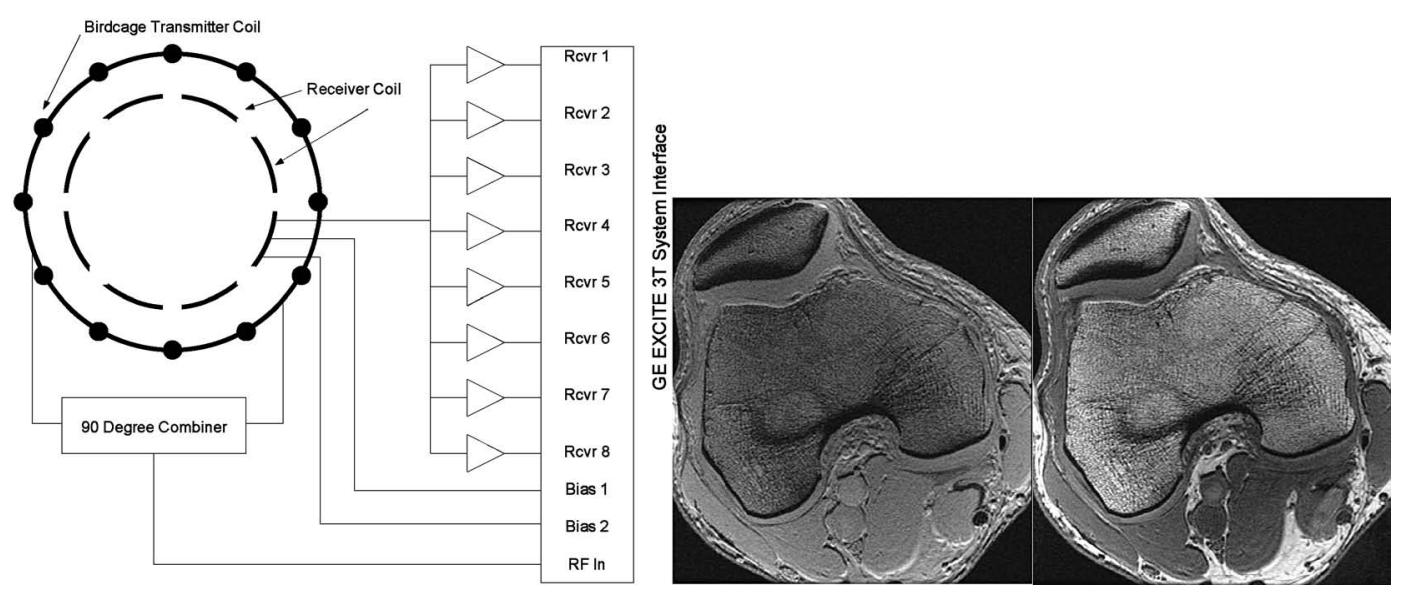

Fig. 7. Illustration of the $3 \mathrm{~T}$ QD birdcage transmit and 8-channel receive hybrid coil and 3T knee images obtained with the coil. (left: 3D SPGR, $\alpha=3^{\circ}$; right: 3D SPGR, $\left.\alpha=18^{\circ}\right)(\mathrm{Im}-$ ages Courtesy Brian Rutt, Robarts Research Institute)

Table 2. The differences between parallel imaging and transmit-SENSE

\begin{tabular}{lll}
\hline & Parallel Imaging & $\begin{array}{c}\text { Transmit-SENSE } \\
\text { (parallel transmission of } \\
\text { multidimensional RF pulses) }\end{array}$ \\
\hline What do we know? & $\begin{array}{l}\text { Aliased images, } \\
\text { coil sensitivities }\end{array}$ & $\begin{array}{l}\text { Full field-of-view excitation, } \\
\text { coil sensitivities }\end{array}$ \\
\hline What do we want to know? & $\begin{array}{l}\text { Full field-of-view } \\
\text { images }\end{array}$ & Aliased excitations \\
\hline Can we use preamplifier decoupling? & Yes & $\begin{array}{l}\text { No } \rightarrow \text { other decoupling } \\
\text { techniques needed }\end{array}$ \\
\hline
\end{tabular}

\section{State-of-the-Art Examples}

One-and-a-half-Tesla MR imaging systems are the gold standard in many clinical applications, and some systems are now available with $96 \mathrm{RF}$ receiver channels. ${ }^{13}$ 3T systems have proven their advantages in brain and musculoskeletal imaging, and rapid development in body applications are in progress. The $3 \mathrm{~T}$ market is being recognized as a growing high-end clinical market, and some 3T systems are now equipped with $128 \mathrm{RF}$ receiver channels (Fig. 6[a]). On the other hand, a few 7T systems are available worldwide that are targeting the study of brain function; functional brain imaging requires a finer spatial scale, with resolutions at a sub-millimeter scale. Researchers are hoping that such brain analysis enables improved treatment of neurodegenerative diseases, such as Alzheimer's disease. Because of the available intrinsic high SNR at $7 \mathrm{~T}$ (Fig. 6[b] and [c]), ${ }^{37}$ images based on 30 or more physicochemical parameters are used to examine tissue morphology, blood flow, metabolism, and chemistry in vivo. Many different RF detector array coils are available at these different static magnetic-field strengths. Figure 6(d) shows an example of shield/transmission-line type designs, referred to as volume-strip arrays. ${ }^{38}$

Nonetheless, the essential questions remain: what are the clinical applications; how many channels/coils do we have available and use; how do we decouple the coils; and how do we optimize the SNR?

Another important consideration is the specific absorption rate, SAR, which is the quantity characterizing the energy deposited into a unit mass: ${ }^{39}$

$$
S A R \propto \omega_{0}^{2} B_{1}^{2} a^{2} \quad \text { Eq. (7), }
$$

where $\omega_{0}=$ Larmor angular frequency, $B_{1}=$ transmit RF magnetic field amplitude, and $a=$ coil diameter (or patient size). As the static magnetic field increases from 1.5 to $3 \mathrm{~T}$, the resultant SAR at $3 \mathrm{~T}$ is 4 times that at $1.5 \mathrm{~T}$. Thus, at higher field strengths, SAR becomes the issue for particular sequences that require a large flip angle and/or many slices to be collected within the repetition time (TR). In such cases, a hybrid coil can be advantageous. A hybrid 
coil consists of a localized dedicated transmitter coil whose size is just optimized for a target ROI/ FOV and an independent set of receiver coils as shown in Fig. $7 .{ }^{40}$ Because the transmitter coil is much smaller than a typical whole body transmitter coil, the localized transmitter requires less input power to generate the necessary $B_{1}$ or a much larger $\mathrm{B}_{1}$ can be generated that translates into a shorter RF pulse duration and potentially reduced SAR. Furthermore, Eq. (7) means that an RF coil with half the diameter yields one fourth the SAR provided that $B_{1}$ remains unchanged. For this reason, the hybrid coil is considered an effective approach at higher static magnetic-field strengths.

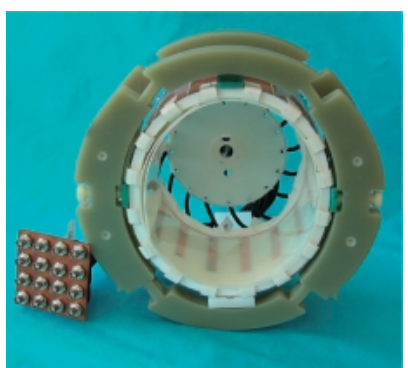

Fig. 8. A $9.4 \mathrm{~T}, 400 \mathrm{MHz}$ $\mathrm{T} / \mathrm{R}$ multi-element TEM parallel transceiver head coil for $\mathrm{B}_{1}$ control. (Courtesy Thomas Vaughan, University of Minnesota)

a
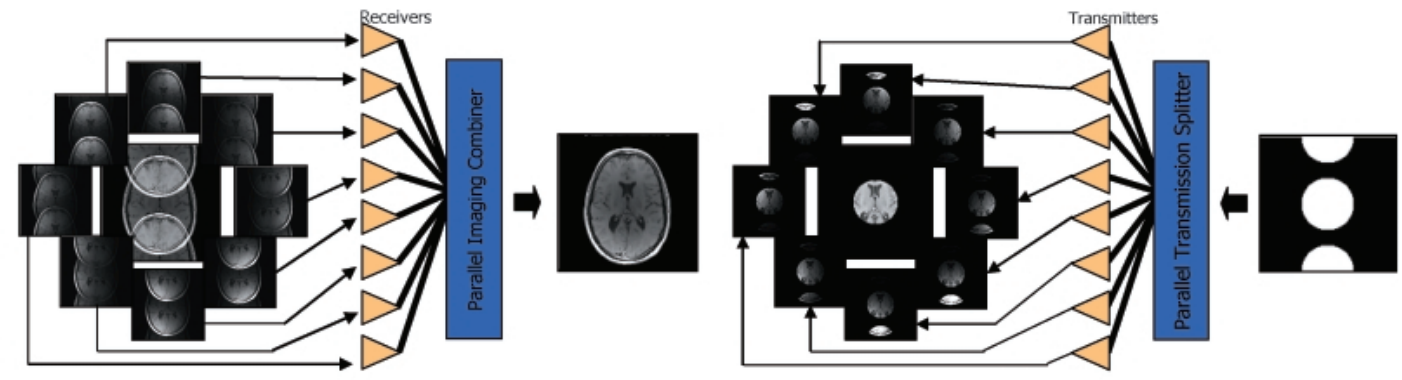

b
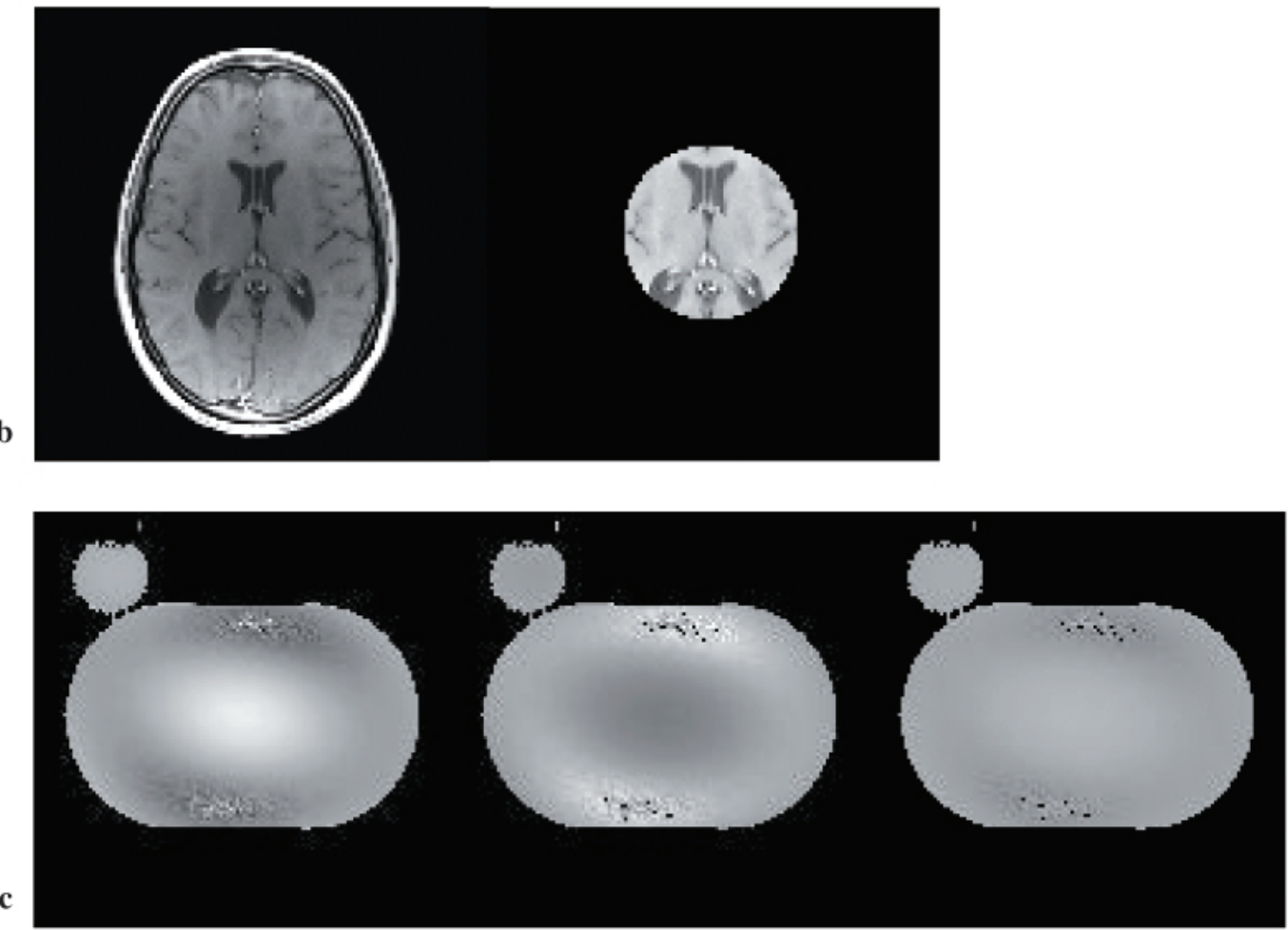

(Intrinsic Field) $\times$ (Input Pulse) $=($ Resultant Field $)$

Fig. 9. (a) Illustration of direction of flow and location of aliased information. (left: parallel imaging; right: Transmit-SENSE). (b) Normal excitation versus inner volume excitation. (c) Example of homogeneity correction. (Courtesy Mark A. Griswold, Case Western Reserve University) 

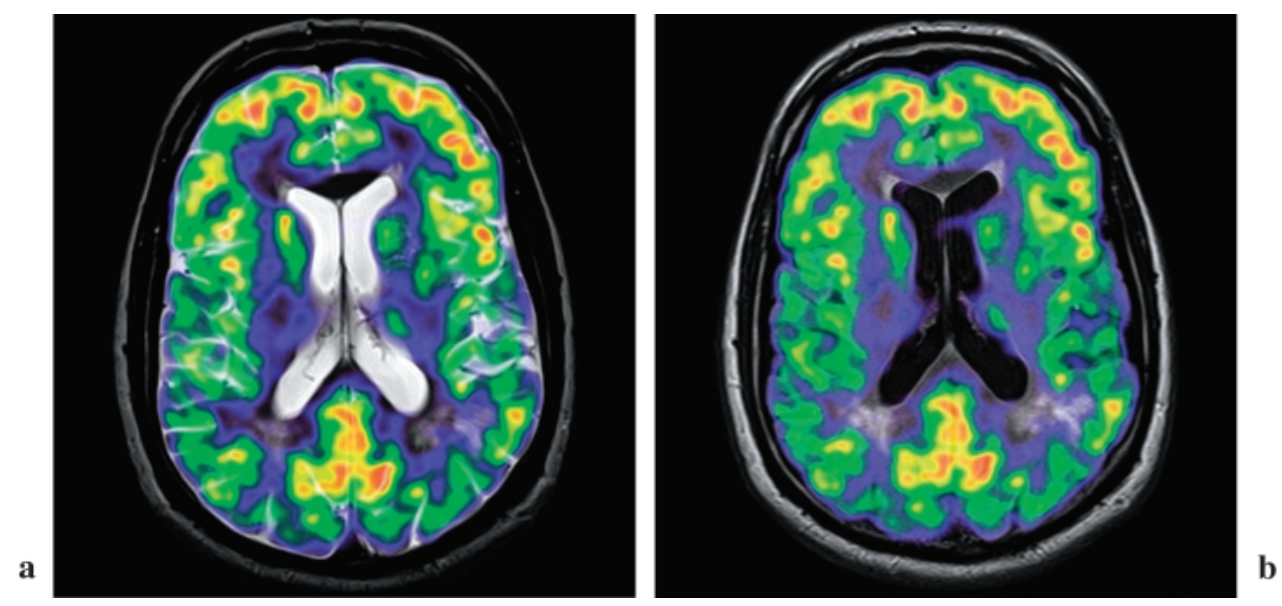

Fig. 10. The world's first simultaneously obtained MR-PET human brain images. The patient was injected with FDG, and the PET acquisition took $20 \mathrm{~min}$. During the PET acquisitions MR images with $\mathrm{T}_{2}$ (a) and Dark Fluid contrast (b) were acquired. The images show the findings: periventricular gliotic foci in the white matter mostly present in the bioccipital region, and calcification-iron deposition of the basal ganglia. (Courtesy Siemens Medical Solutions, University of Tennessee, and University of Tuebingen)

\section{Transmit-SENSE}

At very high frequencies, e.g., 3T and above, wavelength effects within a dielectric object have been reported to produce non-uniformity issues, as seen in dielectric resonance in which object dimension and wavelength coincide. Thus, achieving $\mathrm{B}_{1}$ transmission to yield a uniform $\mathrm{B}_{1}$ field is challenging. This is why some researchers attempt to correct the $\mathrm{B}_{1}$ field by manipulating the magnitudes and phases associated with each transmitter channel, known as "mode-scanning excitation" (Fig. 6[d]) $)^{38}$ and " $B_{1}$ shimming" (Fig. 8). ${ }^{41}$

However, this $B_{1}$ shimming process is an iterative process, and keen insight is required to control $B_{1}$ spatial modulation. Transmit-SENSE, ${ }^{42}$ on the other hand, is an inverse approach. It is well known that a Fourier transform relationship exists in the reception (omitting constants for simplicity) between signal and image (i.e., object):

$$
M(\vec{x})=\int S(k) e^{-i \vec{x} k} d k
$$

Katscher's group realized that, in transmission, there is a Fourier transform relationship between the excitation RF pulse shape and the 3-D excited spatial profile:

$$
M_{\text {excite }}(\overrightarrow{\boldsymbol{x}})=\int B_{1}(k) e^{-i \bar{x} k} d k
$$

In the Transmit-SENSE approach, different timevarying $B_{1}$ pulses in each coil of the array are used to excite a multi-dimensional profile in a reduced time. ${ }^{43}$

Table 2 illustrates the differences between parallel imaging and Transmit-SENSE. With regard to RF coil design, the major difference between SENSE and Transmit-SENSE is that SENSE can utilize preamplifier decoupling among the detector array coil elements, but TransmitSENSE cannot because the current is required to produce a $\mathrm{B}_{1}$ transmit field. Thus, learning how to decouple multiple transmit coil elements still remains a challenge. Kurpad's and Vernickle's groups have presented examples of coil designs for Transmit-SENSE. ${ }^{44,45}$

As recognized in Table 2, with respect to direction of flow and location of aliased information, process flow is opposite for parallel imaging and Transmit-SENSE. The flow is depicted in Fig. 9(a). Use of multi-dimensional RF pulses enables following applications such as inner volume excitation (Fig. 9[b]; faster imaging in localized regions) and homogeneity correction ( $B_{1}$ shimming; Fig. 9[c]). By designing RF pulses to compensate for transmission inhomogeneity and susceptibility correction, which is for $\mathrm{B}_{0}$, inhomogeneity is corrected.

\section{Emerging/Future RF Coil Technologies}

The healthcare industry and medical technologies are rapidly advancing, and each medical specialty may be supported by a large industrial effort. However, because of the variances in function between specialties, it is anticipated that many different medical technologies will be integrated to 
Table 3. Various imaging modalities

\begin{tabular}{|c|c|c|c|c|}
\hline $\begin{array}{l}\text { Imaging } \\
\text { Modality }\end{array}$ & $\begin{array}{l}\text { At what are } \\
\text { we looking? }\end{array}$ & $\begin{array}{l}\text { Ionizing } \\
\text { Radiation }\end{array}$ & Pros & Cons \\
\hline $\begin{array}{l}\text { Computed } \\
\text { Tomography/ } \\
\text { X-ray }\end{array}$ & Electron density & Yes & $\begin{array}{l}\text { Fast scan time, } \\
\text { high spatial } \\
\text { resolution, } \\
\text { widespread } \\
\text { access }\end{array}$ & $\begin{array}{l}\text { Radiation } \\
\text { exposure, } \\
\text { compromised } \\
\text { soft tissue } \\
\text { contrast }\end{array}$ \\
\hline $\begin{array}{l}\text { PET (Nuclear } \\
\text { Medicine*): } \\
\text { imaging with } \\
\text { positron emitters }\end{array}$ & $\begin{array}{l}\text { Injection of a } \\
\text { radioactively } \\
\text { labeled drug that } \\
\text { accumulates in } \\
\text { target organs or } \\
\text { pathologic } \\
\text { tissue, tracers } \\
\text { labeled with } \\
\text { positron emitters }\end{array}$ & $\begin{array}{l}\text { Negligible } \\
\text { (equivalent to } \\
\text { amount we } \\
\text { receive from } \\
\text { natural } \\
\text { environment } \\
\text { each year) }\end{array}$ & $\begin{array}{l}\text { Characterization } \\
\text { of biochemical } \\
\text { functions of } \\
\text { cells, organs, and } \\
\text { body structures } \\
\text { in vivo, } \\
\text { metabolic } \\
\text { activity at a } \\
\text { molecular level }\end{array}$ & $\begin{array}{l}\text { Compromised } \\
\text { spatial resolution } \\
\text { (blurring), about } \\
\text { one hour to wait } \\
\text { for the injection } \\
\text { to go through the } \\
\text { body prior to } \\
\text { imaging }\end{array}$ \\
\hline $\begin{array}{l}\text { Magnetic } \\
\text { Resonance } \\
\text { Imaging }\end{array}$ & $\begin{array}{l}\text { Proton density } \\
\text { (mostly) }\end{array}$ & No & $\begin{array}{l}\text { High spatial } \\
\text { resolution, } \\
\text { excellent soft } \\
\text { tissue contrast }\end{array}$ & $\begin{array}{l}\text { Patients with } \\
\text { pacemakers, } \\
\text { claustrophobia, } \\
\text { acoustic noise }\end{array}$ \\
\hline $\begin{array}{l}\text { SPECT } \\
\text { (Nuclear } \\
\text { Medicine*): } \\
\text { imaging with } \\
\text { single photon } \\
\text { emitters }\end{array}$ & $\begin{array}{l}\text { Radiotracers in } \\
\text { the human body } \\
\text { with various } \\
\text { decay energies }\end{array}$ & Negligible & $\begin{array}{l}\text { Simpler scanner } \\
\text { than PET }\end{array}$ & $\begin{array}{l}\text { Compromised } \\
\text { localization } \\
\text { capability } \\
\text { compared with } \\
\text { PET }\end{array}$ \\
\hline Ultrasound & $\begin{array}{l}\text { Propagation of } \\
\text { sound wave } \\
\text { (f=1 to } 15 \\
\mathrm{MHz} \text { ) into the } \\
\text { patient (back } \\
\text { scattering) }\end{array}$ & No & $\begin{array}{l}\text { Portability, } \\
\text { real-time } \\
\text { imaging }\end{array}$ & $\begin{array}{l}\text { Image quality } \\
\text { too dependent on } \\
\text { specific patient }\end{array}$ \\
\hline
\end{tabular}

*Nuclear medicine involves the emission of radiation from the organ or tissue, whereas radiology involves the transmission of radiation into the tissue.

realize more powerful and useful diagnostic tools. The principle underlying the combination of imaging modalities, such as PET-CT, ${ }^{46}$ is that each provides different image contrasts and gives different information. In this example, because CT reveals the shape of cancerous tissue and PET shows the activity of the cancer, PET and CT together simultaneously reveal information about tumor shape, location, and activity. Siemens Medical Solutions (Erlangen, Germany) is working on combining the strengths of MR (soft tissue contrast) and PET (molecular information), ${ }^{47}$ as reported in Fig. 10. Table 3 summarizes the various imaging modalities. Successful integration requires other modalities to at least be compatible with MR, i.e., non-magnetic, and RF coil designs will have to take the requirements of other modalities into consideration. For instance, Kuroda is working on an MR-compatible endoscope made of titanium, glass, and polymers with a $1.5-\mathrm{cm}$ ID for a tip RF coil. ${ }^{48}$ This yet unknown and emerging field will further constrain the design of MRI RF coils.

The possibility of wireless RF coils is worth mentioning. As discussed earlier and elsewhere, ${ }^{9}$ cabling is a challenge in the design of RF receiver coils with many channels because of couplings among the cables ${ }^{49}$ and coil elements, space limitations, and weight. Obviously, a cable with galvanic interconnections exists between an RF coil and MR imaging system to: send the MRI RF signal with its high dynamic range (typically $150 \mathrm{~dB}$ with modern systems) and bandwidth; control signals 
for tuning/detuning, recognizing coil ID and coil status; and supply power to the onboard components. Wireless technologies may advance safety by eliminating baluns and offering convenience and ease of operation for particular clinical applications (e.g., in the use of the anterior cardiac array coil). On the other hand, challenges include finding methods to maintain the phase information of individual signals..$^{50}$ This can be done in the analog domain by mixing the RF signals on to higher frequency carriers, or it can be done digitally if each coil is equipped with its own A/D converter. Another issue is that the necessary wireless data transmission can be greater than $1 \mathrm{~GB} / \mathrm{s}$ for a highend MR imaging scanner with high receiver-channel count, assuming the required bits accuracy, sampling rate, maximum gradient strength, and FOV. This specification is not yet available from the current WLAN industry. Furthermore, the implementation of wireless technology requires a battery to power the coil and its associated on-board electronics. However, it is anticipated that the aforementioned challenges will be overcome because of the rapid technological advancement in the industry, as evidenced by the growth of the wireless communication industry. Thus, the challenge remains as to how we can take advantage of these emerging technologies.

\section{Summary}

The subject of array coil design is complex and encompasses the entire reception chain and emerging applications of parallel imaging and transmission technologies. Although there are many coil designs for different applications at various $\mathrm{B}_{0}$ field strengths, the principles underlying the design of detector array coils are essentially the same. In particular, preamplifier decoupling in view of a mismatched $\lambda / 4$ matching circuit is important. Design of RF detector array coils must take into consideration that: (1) SNR be optimized; (2) for a given clinical application at a selected $\mathrm{B}_{0}$, the number of available receiver channels, desirable phase encoding direction, target acceleration factor, and FOV determine the coil design of choice; and (3) SNR (for spatial resolution) and speed of acquisition (for temporal resolution, i.e., acceleration factor) are best balanced depending upon clinical application.

Additional integration of different imaging modalities is anticipated, which will further constrain design of RF array coils intended for either reception-only or transmission/reception.

\section{Acknowledgments}

I am grateful to all my friends and colleagues with whom I have interacted professionally. In particular, I would like to thank Dr. Xiaoyu Yang and Tsinghua Zheng at Case Western Reserve University (CWRU) for their invaluable discussions on preamplifier decoupling; Professors Mark A. Griswold (CWRU), Ray F. Lee (New York University), and Larry L. Wald (Massachusetts General Hospital [MGH]) and Drs. Patrick Ledden (Nova Medical), Jeff H. Duyn (National Institutes of Health), Brian Rutt (Robarts Research Institute), J. Tommy Vaughan (University of Minnesota), Ioannis Panagiotelis (Siemens Medical Solutions), Juergen Kampmeier (Siemens Medical Solutions), and John L. Patrick (Philips Medical Systems) for providing their invaluable pictures/images; Professor Robert W. Brown (CWRU) for his invaluable comments and discussions to improve the manuscript; and Matthew Finnerty for his assistance in generating a plot of Fig. 4(b). I am also grateful for discussions with Drs. Johan Overweg (Philips Research Laboratories, Hamburg, Germany), Arne Reykowski (InVivo Corporation), and Ralph Oppelt (Siemens Corporate Research, Germany) at the 2005 ISMRM MR Engineering Study Group Workshop on Wireless MRI Coil Technology and with Wayne Dannels (Toshiba Research Institute USA) that guided my description on the approach to desirable wireless data signal processing. Professors Steve Wright (Texas A \& M University), Stuart Crozier (The University of Queensland), Steve Conolly (Stanford University and University of California, Berkeley), Dr. Hubertus Fischer (Siemens Medical Solutions), and Kazuya Okamoto (Toshiba Medical Systems Corporation) are gratefully acknowledged for their useful discussions over the years. Finally, I would like to thank Professor Katsumi Kose (Tsukuba University), Dr. Fumiyuki Mitsumori (National Institute for Environmental Studies), and Professor Tsuneya Watabe (Editor-inChief, Magnetic Resonance in Medical Sciences) for providing me with this wonderful opportunity to write a review article on this RF coil-related subject.

A part of this work was presented at the Fourteenth Scientific Meeting of the ISMRM ${ }^{51}$ and the $34^{\text {th }}$ Annual Scientific Meeting of the Japanese Society of Magnetic Resonance in Medicine held at the Tsukuba International Conference Hall in $2006 .{ }^{52}$ 


\section{References}

1. Hoult DI. The NMR receiver: a description and analysis of design. Progress in NMR spectroscopy 1978; 12:41-77.

2. Roemer PB, Edelstein WA, Hayes CE, Souza SP, Mueller OM. The NMR phased array. Magn Reson Med 1990; 16:192-225.

3. Hayes CE, Hattes N, Roemer PB. Volume imaging with MR phased arrays. Magn Reson Med 1991; 18:309-319.

4. Fenn AJ, Temme DH, Delaney WP, Courtney WE. The development of phased-array radar technology. Lincoln Lab J 2000; 12:321-340.

5. Stutzman WL, Thiele GA. Antenna theory and design. John Wiley \& Sons, 1981; 108.

6. Wright SM, Wald LL. Theory and application of array coils in MR spectroscopy. NMR Biomed 1997; 10:394-410.

7. Hayes CE, Edelstein WA, Schenck JF, Mueller OM, Eash M. An efficient, highly homogeneous radiofrequency coil for whole-body NMR imaging at 1.5T. J Magn Reson 1985; 63:622-628.

8. Kucera J, Lott U. Low noise amplifier design, In: Golio M, ed. The RF and microwave handbook. CRC Press, 2001; 548-571.

9. Yang X, Zheng T, Fujita H. T/R switches, baluns, and detuning elements in MRI RF coils, In: ISMRM Fourteenth Scientific Meeting Weekend Syllabus 2006.

10. Sodickson DK, Manning WJ. Simultaneous acquisition of spatial harmonics (SMASH): fast imaging with radiofrequency coil arrays. Magn Reson Med 1997; 38:591-603.

11. Pruessmann KP, Weiger $M$, Scheidegger MB, Boesiger P. SENSE: sensitivity encoding for fast MRI. Magn Reson Med 1999; 42:952-962.

12. Griswold MA, Jakob PM, Heidemann RM, et al. Generalized autocalibrating partially parallel acquisitions (GRAPPA). Magn Reson Med 2002; 47: 1202-1210.

13. Wiggins GC, Potthast A, Triantafyllou C, et al. A 96-channel MRI system with 23- and 90-channel phase array head coil at $1.5 \mathrm{~T}$, In: Proceedings of the thirteenth annual meeting of the ISMRM. Miami, 2005; 671.

14. Hoult DI, Richards RE. The signal to noise ratio of the nuclear magnetic resonance. J Magn Reson 1976; 24:71-85.

15. Froncisz W, Jesmanowicz A, Kneeland JB, Hyde JS. Counter rotating current local coils for highresolution magnetic resonance imaging. Magn Reson Med 1986; 3:590-603.

16. Horowitz P, Hill W. In: Chapter 7, The art of electronics. Second edition. Cambridge University Press, 1989; 434-435.

17. Li BK. The design and analysis of high frequency phased array coils for MRI. Ph.D. Thesis. The University of Queensland, 2006; 84-89.

18. Su S, Zou MX, Murphy-Boesch J. Solenoidal array coils. Magn Reson Med 2002; 47:794-799.

19. Jevtic J. Ladder networks for capacitive decoupling in phased-array coils, In: Proceedings of the ninth annual meeting of the ISMRM. Glasgow, $2001 ; 17$.

20. Wang J, Friedrich A. Antenna arrangement for a magnetic resonance apparatus. U.S. Patent 5,708,361.

21. Lee RF, Giaquinto RO, Hardy CJ. Coupling and decoupling theory and its application to the MRI phased array. Magn Reson Med 2002; 48:203-213.

22. Lee RF, Westgate CR, Weiss RG, Newman DC, Bottomley PA. Planar strip array (PSA) for MRI. Magn Reson Med 2001; 45:673-683.

23. Madore B, Pelc NJ. SMASH and SENSE: experimental and numerical comparisons. Magn Reson Med 2001; 45:1103-1111.

24. Herlihy DJ, Larkman DJ, Fujita H, Burl M, Hajnal JV. A 4-channel head coil for SENSE imaging, In: Proceedings of the eighth annual meeting of the ISMRM. Denver, 2000; 1394.

25. Hajnal JV, Larkman DJ, Herlihy DJ. An array that exploits phase for SENSE imaging, In: Proceedings of the eighth annual meeting of the ISMRM. Denver, 2000; 1719.

26. Fujita H, Spence DK. A novel 8-channel "saddletrain", array coil for abdominal SENSE imaging at 1.5T, In: Proceedings of the 10th annual meeting of the ISMRM. Hawaii, 2002; 326.

27. Boskamp E, Blawat L, King KF, Lorbiecki J, Tiwari AB. A 16-channel neurovascular SENSE array, In: Proceedings of the tenth annual meeting of the ISMRM. Hawaii, 2002; 852.

28. Blawat LR, Hoppel BE, Boskamp EB, Iwadate Y. 3-T breast array optimized for parallel imaging, In: Proceedings of the twelfth annual meeting of the ISMRM. Kyoto, 2004; 1596.

29. Okamoto K, Takagi M, Ishii $\mathrm{M}$, et al. A novel "smart" neurovascular array coil system for parallel imaging, In: Proceedings of the twelfth annual meeting of the ISMRM. Kyoto, 2004; 1595.

30. Ohliger MA, Greenman RL, Giaquinto R, McKenzie CA, Wiggins G, Sodickson DK. Concentric coil arrays for parallel MRI. Magn Reson Med 2005; 54:1248-1260.

31. Spencer D, Akao J, Duensing R, et al. Design of a 32-channel cardiac array for parallel imaging, In: Proceedings of the thirteenth annual meeting of the ISMRM. Miami, 2005; 911.

32. Lanz T, Kellman, Nittka M, Greiser A, Griswold M. A 32-channel cardiac array optimized for parallel imaging, In: Proceedings of the fourteenth annual meeting of the ISMRM. Seattle, 2006; 2578.

33. Reykowski A, Hemmerlein M, Fischer H. 16-channel head/neck matrix coils for 3 Tesla, In: Proceedings of the thirteenth annual meeting of the ISMRM. Miami, 2005; 908.

34. Weiger M, Pruessmann KP, Leussler C, Roschmann P, Boesiger P. Specific coil design for 
SENSE: a six-element cardiac array. Magn Reson Med 2001; 45:495-504.

35. Ohliger MA, Grant AK, Sodickson DK. Ultimate intrinsic signal-to-noise ratio for parallel MRI: electromagnetic field considerations. Magn Reson Med 2003; 50: 1018-1030.

36. Wiesinger F, De Zanche N, Pruessmann KP. Approaching ultimate SNR with finite coil arrays, In: Proceedings of the thirteenth annual meeting of the ISMRM. Miami, 2005; 672.

37. Ledden PJ, Mareyam A, Wang S, van Gelderen P, Duyn JH. 24-channel receive-only array for brain imaging at 7T, In: Proceedings of the fourteenth annual meeting of the ISMRM. Seattle, 2006; 422.

38. Lee RF, Brown R, Mizsei G, et al. Implementation of mode-scanning excitation method with a 16channel transmit/receive volume strip array at $7 \mathrm{~T}$, In: Proceedings of the fourteenth annual meeting of the ISMRM. Seattle, 2006; 125.

39. Haacke EM, Brown RW, Thompson MR, Venkatesan R. Magnetic resonance imaging: physical principles and sequence design. New York: Wiley-Liss, 1999; 858-859.

40. Yang X, Fujita H, Zheng T, Richmond K, Colwell C. A 3-T QD transmit/8-channel receive hybrid knee coil, In: Proceedings of the thirteenth annual meeting of the ISMRM. Miami, 2005; 907.

41. Vaughan J, DelaBarre L, Snyder C, et al. 9.4-T human imaging: preliminary results, In: Proceedings of the fourteenth annual meeting of the ISMRM. Seattle, 2006; 529.

42. Katscher U, Boernert P, Leussler C, van den Brink JS. Transmit SENSE. Magn Reson Med 2003; 49:144-150.
43. Zhu Y. Parallel excitation with an array of transmit coils. Magn Reson Med 2004; 51:775-784.

44. Kurpad KN, Boskapm EB, Balanis SM. A parallel transmit volume coil with independent control of currents on the array elements, In: Proceedings of the thirteenth annual meeting of the ISMRM. Miami, 2005; 16.

45. Vernickle $\mathrm{P}$, Roschmann $\mathrm{P}$, Findeklee C, et al. An 8-channel transmit/receive body coil for 3T, In: Proceedings of the foureenth annual meeting of the ISMRM. Seattle, 2006; 123.

46. Arnulf Oppelt, ed. Imaging systems for medical diagnostics. Erlangen: Publicis Corporate Publishing, 2005; 830-835.

47. Fischer H, Ladebeck R, Schmand MJ. Challenges and technical solutions of a combined MR PET scanner. Jpn J Magn Reson Med 2006; 26 (Suppl): 111.

48. Kuroda K. MR compatible endoscope. Nihon Keizai Shinbun 2005; 18 November.

49. Koste GP, Nielsen MC, Tolliver TR, Frey RL, Watkins RD. Optical MR receive coil array interconnect, In: Proceedings of the thirteenth annual meeting of the ISMRM. Miami, 2005; 411.

50. Scott G, Yu K. Wireless transponders for RF coils: systems issues, In: Proceedings of the thirteenth annual meeting of the ISMRM. Miami, 2005; 330.

51. Fujita H, Yang X, Zheng T. Array coil types and design principles, In: ISMRM Fourteenth Scientific Meeting Weekend Syllabus 2006.

52. Fujita H. New horizons in MR technology: from a perspective of RF coil designs and trends. Jpn J Magn Reson Med 2006; 26 (Suppl): 111. 